\title{
Integrated Approach to Conceptual Modeling
}

\author{
Lindita Nebiu Hyseni \\ Faculty of Contemporary Sciences and Technologies \\ South East European University \\ Tetovo, Macedonia
}

\author{
Zamir Dika \\ Faculty of Contemporary Sciences and Technologies \\ South East European University \\ Tetovo, Macedonia
}

\begin{abstract}
Conceptual modeling is supporting understanding and communication of the requirements in developing the information system (IS). The nature of requirements is usually divided into the functional (FRs) and non-functional requirements (NFRs). Thus, the scholars who are representing the conceptual modeling separate FRs and NFRs. Attempting to create an integrated framework for conceptual modeling of FRs and NFRs a new approach is being represented. In order to justify the approach, the research work and relevant literature in the field by using integrated perspective has been analyzed in this paper. As an outcome of this review, the need for an integrated approach to the requirement determination and proposed an integrated framework for conceptual modeling, which shall include the functional and non-functional requirements in one conceptual model has been identified. There is also identified that a small number of researchers have worked in this field, whilst the failure rate of the IS implementation which continues to be high has been the motivation, therefore the concept of proposing an integrated framework which will contribute to the increase of the efficacy of the requirements from the analysis phase in order to secure the sustainability of the IS has been approached.
\end{abstract}

Keywords-Integrated Framework; Conceptual Model; Functional Requirements; Non-Functional Requirements; Research Gaps; Joint Approval Requirements (JAR)

\section{INTRODUCTION}

The information system (IS) is an integrated set of the Information and Communication Technology (ICT) components, human resources and processes which supports individuals, groups, organizations and society in achieving their business and societal goals. Therefore, it is very important to support communication and understanding of the requirements in the development of the information system by using the conceptual model. The conceptual model is the model of the information system represented in a simple way in order to be understood by all participants in the project team and to be developed faster in fulfilling the requirements during the knowledge acquisition [1]. Conceptual model plays crucial role in the success of the information system [2], [3], [4]. After proven that the success of conceptual model affects the success of the information system, is asked the research question, what is needed to improve the conceptual modeling? Based on this research questions, the focus turns to the nature of the functional and non-functional requirements and their integration in one conceptual model, source used for the conceptual modeling, quality applied in the conceptual modeling, instrument used to design the conceptual model and method which is used by the client for approval of the requirements and conceptual model.
System requirements importance in developing the successful information system is also treated by scholars in 2016, who showed that participants involved in the project of the information system, start to clarify incompleteness and inconsistencies only during the phase of the system implementation but not at the phase of the system requirements, thus having an impact on the failure rate of the information system [5], [6]. Accordingly, is needed to conduct research by identifying if the scholars have started to initiate the integration of the functional and non-functional requirements in one conceptual model. Based on this, the objective in this paper is to present the rationale behind the proposal for integration of functional and non-functional requirements in one conceptual model, through a state of the art review for the identification of the research gap. This paper is organized as follows: in the section 2 is state of the art; in the section 3 are presented identified research gaps in the conceptual modeling; in the section 4 is presented the proposed integrated framework for conceptual modeling; in the section 5 is conclusion and future work; at the end, in the section 6 are presented the references used in this paper.

\section{STATE OF THE ART}

Based on the literature reviewed, development of the conceptual model is treated from different research outcomes, but in this section, are presented researches relevant to the functional and non-functional requirements.

\section{A. Conceptual model}

During the research conducted it is identified that the researchers nowadays have initiated to consider the nonfunctional requirements during the information system development. The authors Zubcoff, Garrigos, Casteleyn, Mazon, and Aguilar (2016), presented the Pareto method in which they considered the non-functional requirements during analysis and modeling of the requirements, in order to increase the quality of the web application, improve the design decision accuracy during the requirements analysis phase and they also stated the possibility for reducing the time needed by the designers [7]. Even though the issue of the non-functional requirements is initiated by above mentioned authors; Afreen, Khatoon and Sadiq (2016) in their article highlighted that most of the work is related to the functional requirements, while the non-functional requirements have received less consideration by the goal-oriented requirements engineering community [8]. In the article proposed by Neto, Vargas-Solar, da Costa, and Musicante (2016) it is also concluded that the non-functional requirements are not considered sufficiently in all software engineering phases [9]. The above articles treated the issue of 
consideration of the non-functional requirements during information system modeling, but they did not introduce the method how to integrate the non-functional requirements. A hint about the possibility of the integration of the functional and non-functional requirements is presented by the authors Eckhardt, Vogelsang, and Fernández (2016), who presented the differences between FRs \& NFRs whilst their results suggested that many non-functional requirements can be handled similarly to functional requirements [10]. This opens the possibility to propose a solution for consideration of the nonfunctional requirements and functional requirements during the conceptual modeling in order to closely resemble the conceptual model of the information system with the real world.

Most of the research articles about the conceptual modeling are not making any distinction, nor putting the argumentation about the effects of the conceptual model versus functional or non-functional requirements. Therefore, the following articles define what type of the requirements belong to conceptual model; two of the articles presented by authors Fatwanto and Boughton (2008) treated the functional and non-functional requirements in separate conceptual models. They have proposed a method to analyze, specify and develop the conceptual models of functional requirements, especially in the context of the translative model-driven development. Whereas, the other article proposes a method to analyze, specify and develop conceptual models of non-functional requirements. They validated these methods by using a case study of the Voter Tracking System [11], [12]. Also, the article presented by Cysneiros and Leite (2004) tackled the issue of the nonfunctional requirements not being treated as first class requirements. In the article, they presented a process to elicit, analyze and trace the non-functional requirements to the functional conceptual model. They also proposed a UML extension in order to allow non-functional requirements to be expressed. Their proposal is tested by three case studies but the results suggested that their proposal can improve quality of the conceptual model. The strategy used by them has some problems in the functional model and its impact. It is not significant in the overall development process [13]. The development process of the information system was also treated in early 1986 by the author Borgida (1986), when he introduced the paper about conceptual modeling of the information system. In this paper the author stated that the information system development can easily be done and used in the natural way by concentrating on the semantic of the application domain [14]. Unlike the author Borgida (1986), Bogumiła, Zbigniew, Lech and Iwona (2016) have presented a method for development of domain models based on knowledge represented by domain ontology [15]. As seen in the last two articles, during the period 1986-2016 is dealt with semantic of application domain and development of domain models.

\section{B. Designing the conceptual model}

In designing the conceptual model, it is very important the source used for modeling. Based on the researches it is noticed that small number of articles related to the guides for documentation of the functional and non-functional requirements are published. This documentation is used as a source for conceptual modeling. Despite the small number of the articles published, contribution to the requirements engineering is high. One of the relevant article is presented by Sommerville, I., \& Sawyer, P. (1997), in which they presented a practical guide to the functional requirements documentation which contains what system should do and is applicable to any type of the information system [16]. Another guide for the functional requirements documentation was presented by authors Gorschek, T., \& Wohlin, C. (2006), who presented the Requirements Abstraction Model (RAM) which enables prioritizing and packaging the requirements in the document before the development of the information system. This document contains what system should do but not how the system should do [17]. That the functional requirements document should contain what system should do, was also agreed by the authors Sommerville, I., \& Sawyer, P. (2015). The authors in their article presented a guide for functional and non-functional requirements, where they divided these two types of requirements in two separated documents, one document for the functional requirements which contains what the system should do and the other document for the nonfunctional requirements [18].

The other important element in designing the conceptual model is the quality in the conceptual modeling, which based on this research is treated by different scholars. The authors Siau and Tan (2005) used the cognitive mapping techniques regarding the quality in the conceptual modeling [19]. While the authors Nelson, Poels, Genero, and Piattini (2012) regarding the quality in the conceptual modeling presented the Conceptual Modeling Quality Framework, when they brought together the framework of Lindland, Sindre, and Sølvberg (LSS) and the one of the Wand and Weber based on Bunge's ontology (BWW) [20]. Unlike the above authors that presented techniques and frameworks, the following authors presented guides which should be considered in order to apply quality during the conceptual modeling. The authors Alencar, Marín, Giachetti, Pastor, Castro, and Pimentel (2009) proposed a guide to solve the gap between specifications of the requirements and the final software products by integrating the Goal-Oriented Requirements Engineering (GORE) and the Model Driven Development (MDD). This guide was proposed for the transformation of the initial requirements into automatically generated software [21]. Another helpful guide presented by authors Silva, Pinheiro, Albuquerque, and Barroso (2016), aims to help the analyst in identifying the functional and non-functional requirements in the contexts of the customer software. In their article, they emphasized that the elicitation of the functional and non-functional requirements is a hard task and time consuming [22]. Unlike the above authors, Andreas, J., \& Frank, T. (2016) treated quality in conceptual modeling by presenting the highlights which are provided by conceptual models [23]. Based on his article the conceptual models provide blueprints for a reasonable, good design of the information system and underlying organizational settings. He also highlighted the IS researchers assume that the application of conceptual model allows time and cost savings and increases the quality.

Moreover, the instrument for designing the conceptual model is very important in the conceptual modeling. Based on 
the research conducted, a small number of articles which treated the instruments for designing the conceptual model is published. The author Wohed (2000) presented his article by introducing an instrument for the complex modern information system jointly with the requirements for shorter development time [24]. Also, the authors Lu and Parsons (2013) presented the instrument called a UML CASE tool for constructing of the UML diagrams which reflect the real world by using the welldefined ontological rules proposed by Evermann and Wand [25]. Another relevant article is presented by authors Ribeiro, Pereira, Rettberg, and Soares (2016), who treated the combination of the UML with the other instruments as SysML and MARTE in order to do modeling of the hardware and software requirements of real-time-systems. The UML and SysML are used for modeling of the functional requirements, while the MARTE is used for modeling of the non-functional requirements. This article also defines which type of requirements can be modeled by which instrument [26]. An instrument which differs from the above instruments due to the cloud computing technology used, is a novel, web-and-based, collaborative, and scalable (meta) modeling tool which supports designing of the domain specific modeling languages and creation of the corresponding domain models. This instrument was presented by authors Maróti, Kecskés, Kereskényi, Broll, Völgyesi, Jurácz, and Lédeczi (2014) [27].

During the conducted literature review it is identified that most of the authors have treated more the impact of cloud computing at system analysis and design giving less importance to the instruments which are used to design conceptual model. The authors Soon and David (2010) presented their article about the impact of the cloud computing on system analysis and design, where they found no differences in the phase of analysis for creating a Cloud based solution, but in the design stage they highlighted the challenges to the database schema, queues, access control, workflow, service bus and query efficiency [28]. The impact of the cloud computing technology is also treated by authors Agarwal and Dhar (2014), who presented the critical issues in predictive modeling for big data and data science [29].

\section{Requirements approval}

The conceptual model is the representation of the information system. Therefore, it is necessary to be reviewed and approved by all participants in the project team from the client side in order for the information system to be completed within time, scope, budget and quality. Based on literature review, is identified that most of the scholars' work was focused on the requirements determination during the session with participants from the client side, even though they have started to initiate the requirements validation but not requirements approval. One of the techniques presented by Bentley, L. D., \& Jeffrey, L. (2007) mentioned requirements validation as optional during the session with participants from the client side. This technique is called Joint Requirements Planning (JRP) and it is for identification, analysis and definition of the requirements during the JRP session. It also includes JRP session planning and JRP session conducting. Benefits of using this technique are that actively involves users and management in the project development, decreases time for finding facts in planning and analysis phase, confirms requirements and approves prototypes if available, produces a formal written document which is published immediately following the JRP session in order to maintain the momentum of the JRP session [30].

The other technique for the requirements definition, but not for the requirements validation or approval, is presented by Dennis, A., Wixom, B. H., \& Roth, R. M. (2012). This technique is called Joint Application Development (JAD) and allows project team, users and management to identify requirements for the information system. It specifies the way of selecting participants in JAD session, the location for the JAD session, the way how to design, prepare and conduct the JAD session and what will JAD post-session report consist of. This JAD technique is used in the analysis and design phase [31].

If is compared the layout of the session room of JRP and JAD technique, the room of the JRP session presents how the room is arranged and the way of the organization of the session by showing in which place is each participant positioned, while the room of the JAD meeting presents only how the room is arranged. Both techniques use "U" shape meeting room.

\section{The summary table}

In the following Table 1 is presented the summary of the state of the art section, where research for conceptual modeling and its concepts is conducted in the period of 1986 to 2016. 
TABLE I. The Summary TABLE OF StATE OF THE ART

\begin{tabular}{|c|c|c|c|c|c|c|c|c|c|c|c|c|c|c|c|c|}
\hline \multirow{2}{*}{ State of the Art } & \multirow{2}{*}{ Concepts } & \multicolumn{15}{|c|}{ Years of the published articles } \\
\hline & & $\stackrel{b}{a}$ & $\stackrel{n}{2}$ & $\underset{\pi}{*}$ & $\stackrel{m}{\approx}$ & 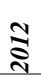 & $\stackrel{2}{\sqrt{2}}$ & 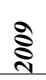 & $\stackrel{\infty}{\stackrel{\sim}{\sim}}$ & ָั & $\widehat{\widehat{\overbrace{}}}$ & $\stackrel{2}{2}$ & 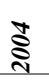 & $\underset{\overbrace{}}{\overparen{ก}}$ & $\hat{\widehat{a}}$ & $\stackrel{\infty}{2}$ \\
\hline \multirow{4}{*}{$\begin{array}{l}\text { Conceptual } \\
\text { model }\end{array}$} & Functional requirements & $\mathrm{x}$ & & & & & & & $\mathrm{x}$ & & & & & & & \\
\hline & Non-functional requirements & $\mathrm{x}$ & & & & & & & $\mathrm{x}$ & & & & & & & \\
\hline & $\begin{array}{l}\text { Integration of the functional and non- } \\
\text { functional requirements }\end{array}$ & $\mathrm{x}$ & & & & & & & & & & & $\mathrm{X}$ & & & \\
\hline & Undefined type of the requirements & $\mathrm{x}$ & & & & & & & & & & & & & & $\mathrm{x}$ \\
\hline \multirow{4}{*}{$\begin{array}{l}\text { Designing the } \\
\text { conceptual } \\
\text { model }\end{array}$} & $\begin{array}{l}\text { Guides for documentation of FRs \& NFRs } \\
\text { in separate documents }\end{array}$ & & $\mathrm{X}$ & & & & & & & $\mathrm{x}$ & & & & & $\mathrm{X}$ & \\
\hline & Quality in the conceptual modeling & $\mathrm{x}$ & & & & $\mathrm{x}$ & & $\mathrm{x}$ & & & & $\mathrm{x}$ & & & & \\
\hline & $\begin{array}{l}\text { Instruments for designing the conceptual } \\
\text { model }\end{array}$ & $\mathrm{x}$ & & $\mathrm{X}$ & $\mathrm{x}$ & & & & & & & & & $\mathrm{X}$ & & \\
\hline & $\begin{array}{l}\text { The impact of the cloud computing on } \\
\text { system analysis and design }\end{array}$ & & & & & & $\mathrm{X}$ & & & & & & & & & \\
\hline \multirow{2}{*}{$\begin{array}{l}\text { Requirements } \\
\text { approval }\end{array}$} & $\begin{array}{l}\text { Joint Requirements Planning (JRP) } \\
\text { Technique }\end{array}$ & & & & & & & & & & $\mathrm{x}$ & & & & & \\
\hline & $\begin{array}{l}\text { Joint Application Development } \\
\text { (JAD)Technique }\end{array}$ & & & & & $\mathrm{X}$ & & & & & & & & & & \\
\hline
\end{tabular}

a. The symbol $\mathrm{x}$ shows published articles in the period 1986-2016 for the listed concepts

As shown in the table 1, in the period 1997-2016 most of the articles have treated the concept of the guide for documentation of the functional and non-functional requirements in separate documents, the quality in the conceptual modeling and the instrument for designing the conceptual model. While, the other concepts of conceptual modeling, which are presented in this table are treated in the period 1986-2016 from a small number of articles out of which most of the articles are during 2016. Considering this, the motivation is triggered to continue the research by identifying research gaps and propose solution based on an integrated approach to conceptual modeling.

\section{RESEARCH GAPS IN THE CONCEPTUAL MODELING}

In this section are summarized gaps identified from the conducted literature review based on the integrated approach to conceptual modeling. As it is presented in the state of the art section, the scholars in the year 2016 have started to treat the integration of the functional and non-functional requirements as a problem, but they did not suggest any solution. However, one scholar hint the possibility of the integration of the functional and non-functional requirements by highlighting that non-functional requirements can be handled similarly to the functional requirements. Furthermore, in 2004 some of the scholars have given a solution to the integration of the functional and non-functional requirements but their result was not satisfactory since there were several problems in their strategy and the impact of the overall development process was not significant. The missing integration of the functional and non-functional requirements in one conceptual model is the main research gap in the field of conceptual modeling. The research gaps identified based on the research conducted which are related to the main research gap are the missing guide for documentation of the functional and non-functional requirements in a document, the missing guide which should be considered to apply quality during conceptual modeling, a missing adequate instrument which supports designing the functional and non-functional requirements in one conceptual model and which works on-premises and on cloud computing technology and a missing method for approval of the client requirements during the session. This method shall include the missing elements in JRP and JAD technique.

Based on these identified research gaps while reviewing literature, integrated framework for conceptual modeling is proposed in the section 4 which shall integrate both, the functional and non-functional requirements in a conceptual model.

\section{THE PROPOSED INTEGRATED FRAMEWORK}

The following algorithm of the proposed integrated framework for conceptual modeling is an outcome of the literature review, identified research gaps and work experience. The aim of this integrated framework is increase of quality of the system requirements and the information system. 


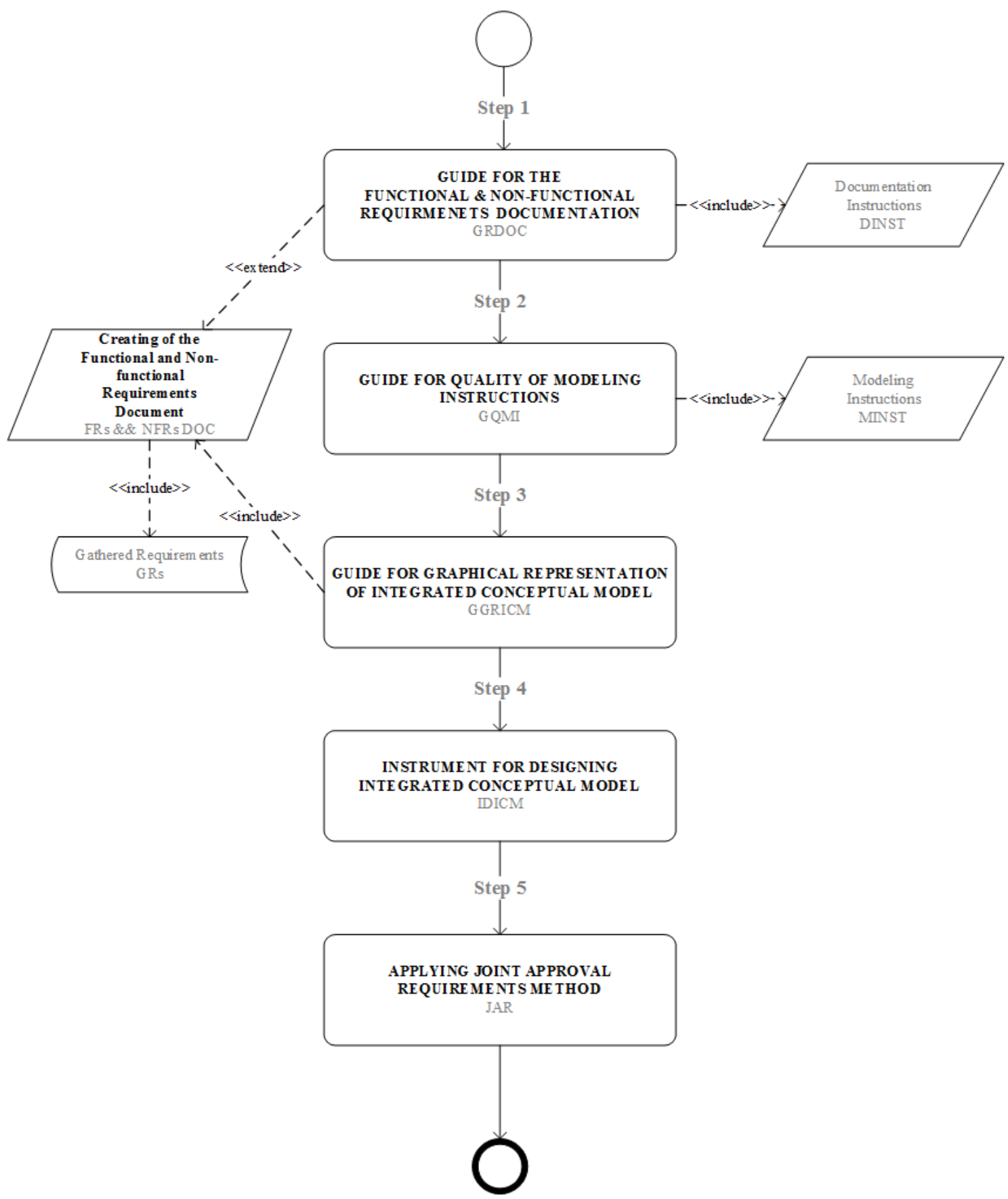

Fig. 1. The algorithm of the Integrated Framework for Conceptual Modeling (IFCMod)

In the Fig. 1, is presented the algorithm of the proposed integrated framework for conceptual modeling (IFCMod) which consists five steps, in the first step is proposed the guide for the functional and non-functional requirements documentation (GRDOC) which should be considered during creation of the requirements document (FRs \& NFRs DOC) based on the documentation instructions (DINST) proposed in this guide and on the gathered requirements (GRs) from documents and texts, the analyst's impressions and reactions, observations, interviews and questionnaires, in the second step is proposed the guide for quality of modeling instructions
(GQMI) which shall be used to apply the quality in the integrated conceptual modeling by using the modeling instruction (MINST), in the third step is proposed the guide for graphical representation of integrated conceptual model (GGRICM) which shows the way of modeling based on proposed guide for quality of modeling instruction (GQMI) and the requirements document (FRs \& NFRs DOC), in the fourth step is the proposed instrument for designing integrated conceptual model (IDICM), and in the fifth step is the method called Joint Approval Requirements (JAR) which is for review and approve the requirements by client in the JAR meetings. 
Based on the Fig. 1, is proposed also the model of the integrated framework for conceptual modeling (IFCMod), which is shown in the Fig. 2.

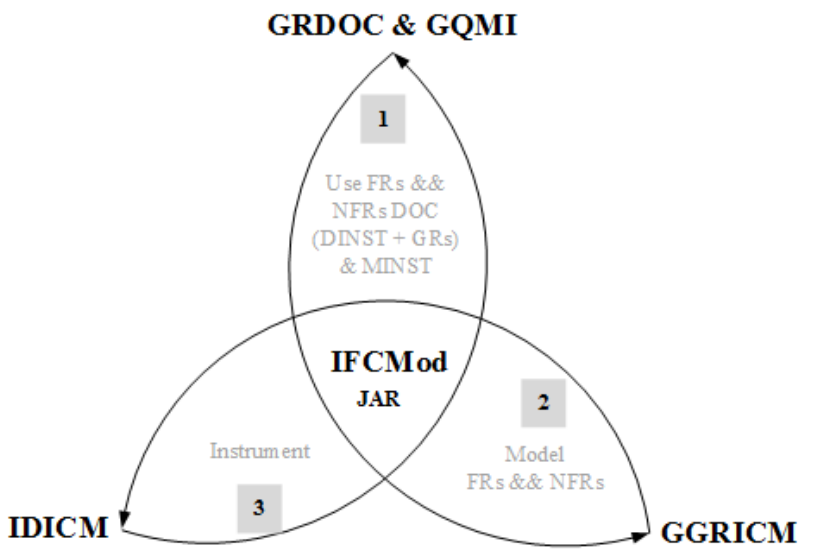

Fig. 2. Conceptual model of IFCMod

The research questions derived from the study about conceptual modeling are presented in the following. Is it possible to create the proposed integrated framework for conceptual modeling (IFCMod)? What will be the documentation instruction (DINST) in the proposed guide for the functional and non-functional requirements documentation (GRDOC) and what will be the impact of this guide in document creation for the functional and non-functional requirements (FRs \& NFRs DOC)? Does have impacted the gathered requirements (GRs) in creating of the functional and non-functional requirements documentation (FRs \& NFRs DOC)? What will be the modeling instruction (MINST) in the proposed guide for quality of modeling instructions (GQMI) and what will be the impact of this guide in the modeling of the integrated conceptual model? Does have impact those two type of guides (GRDOC \& GQMI) in the guide for graphical representation of integrated conceptual model (GGRICM)? What will be the proposed instrument for designing integrated conceptual model (IDICM), will it be flexible to work onpremises and cloud computing technology? Is it possible to create the Joint Approval Requirements (JAR) method? Does have impact the proposed integrated framework for conceptual modeling (IFCMod) in the quality of the system requirements and information system?

\section{THE CONCLUSIONS AND FUTURE WORK}

Based on the identified research gaps through the literature review and work experience, the integrated framework for conceptual modeling (IFCMod) is proposed in this paper. This integrated framework consists of guide for the functional and non-functional requirements documentation (GRDOC), the guide for quality of modeling instructions (GQMI), the guide for graphical representation of integrated conceptual model (GGRICM), the proposed instrument for designing integrated conceptual model (IDICM) and the method called Joint Approval Requirements (JAR). Thus, in the near future, the intention is to continue working on this integrated framework for conceptual modeling (IFCMod) by answering the research questions related to.

\section{REFERENCES}

[1] Robinson, S. (2012, December). Tutorial: Choosing what to modelConceptual modeling for simulation. In Simulation Conference (WSC), Proceedings of the 2012 Winter (pp. 1-12). IEEE.

[2] Bera, P., Krasnoperova, A., \& Wand, Y. (2011). Using ontology languages for conceptual modeling. Cross-Disciplinary Models and Applications of Database Management: Advancing Approaches: Advancing Approaches, 1.

[3] Braga, B. F., Almeida, J. P. A., Guizzardi, G., \& Benevides, A. B. (2010). Transforming OntoUML into Alloy: towards conceptual model validation using a lightweight formal method. Innovations in Systems and Software Engineering, 6(1-2), 55-63.

[4] Mehmood, K., Cherfi, S. S. S., \& Comyn-Wattiau, I. (2010, September). CM-Quality: a pattern-based method and tool for conceptual modeling evaluation and improvement. In Advances in Databases and Information Systems (pp. 406-420). Springer Berlin Heidelberg.

[5] Brinkkemper, S. (2016, March). Gamified Requirements Engineering: Model and Experimentation. In Requirements Engineering: Foundation for Software Quality: 22nd International Working Conference, REFSQ 2016, Gothenburg, Sweden, March 14-17, 2016, Proceedings (Vol. 9619 , p. 171). Springer.

[6] Wagner, D. N. (2016). Breakin'the Project Wave: Understanding and avoiding failure in project management.

[7] Zubcoff, J. J., Garrigos, I., Casteleyn, S., Mazon, J. N., \& Aguilar, J. A. (2016). Evaluating the use of Pareto Efficiency to Optimize NonFunctional Requirements Satisfaction in i* Modeling. IEEE Latin America Transactions, 14(1), 331-338.

[8] Afreen, N., Khatoon, A., \& Sadiq, M. (2016). A Taxonomy of Software's Non-functional Requirements. In Proceedings of the Second International Conference on Computer and Communication Technologies (pp. 47-53). Springer India.

[9] Neto, P. A. S., Vargas-Solar, G., da Costa, U. S., \& Musicante, M. A. (2016). Designing service-based applications in the presence of nonfunctional properties: A mapping study. Information and Software Technology, 69, 84-105.

[10] Eckhardt, J., Vogelsang, A., \& Fernández, D. M. (2016, May). Are nonfunctional requirements really non-functional: an investigation of nonfunctional requirements in practice. In Proceedings of the 38th International Conference on Software Engineering (pp. 832-842). ACM.

[11] Fatwanto, A., \& Boughton, C. (2008, December). Analysis, Specification and Modeling of Functional Requirements for Translative Model-Driven Development. In Knowledge Acquisition and Modeling, 2008. KAM'08. International Symposium on (pp. 859-863). IEEE.

[12] Fatwanto, A., \& Boughton, C. (2008, December). Analysis, Specification and Modeling of Non-Functional Requirements for Translative Model-Driven Development. In Computational Intelligence and Security, 2008. CIS'08. International Conference on (Vol. 2, pp. 405-410). IEEE.

[13] Cysneiros, L. M., \& Leite, J. C. S. D. P. (2004). Nonfunctional requirements: From elicitation to conceptual model. Software Engineering, IEEE Transactions on, 30(5), 328-350.

[14] Borgida, A. (1986). Conceptual modeling of information systems. In on Knowledge Base Management Systems (pp. 461-469). Springer New York.

[15] Bogumiła, H., Zbigniew, H., Lech, T., \& Iwona, D. (2016). Conceptual Modeling Using Knowledge of Domain Ontology. In Intelligent Information and Database Systems (pp. 554-564). Springer Berlin Heidelberg.

[16] Sommerville, I., \& Sawyer, P. (1997). Requirements engineering: a good practice guide. John Wiley \& Sons, Inc.

[17] Gorschek, T., \& Wohlin, C. (2006). Requirements abstraction model. Requirements Engineering, 11(1), 79-101.

[18] Sommerville, I., \& Sawyer, P. (2015). Requirements engineering (10th Edition). Pearson.

[19] Siau, K., \& Tan, X. (2005). Improving the quality of conceptual modeling using cognitive mapping techniques. Data \& Knowledge Engineering, 55(3), 343-365. 
[20] Nelson, H. J., Poels, G., Genero, M., \& Piattini, M. (2012). A conceptual modeling quality framework. Software Quality Journal, 20(1), 201-228.

[21] Alencar, F., Marín, B., Giachetti, G., Pastor, O., Castro, J., \& Pimentel, J. H. (2009). From i* requirements models to conceptual model of a model driven development process. In The Practice of Enterprise Modeling (pp. 99-114). Springer Berlin Heidelberg.

[22] Silva, A., Pinheiro, P., Albuquerque, A., \& Barroso, J. (2016). A Process for Creating the Elicitation Guide of Non-functional Requirements. In Software Engineering Perspectives and Application in Intelligent Systems (pp. 293-302). Springer International Publishing.

[23] Andreas, J., \& Frank, T. (2016). Towards a document-driven approach for designing reference models: From a conceptual process model to its application. Journal of Systems and Software, 111, 254-269.

[24] Wohed, P. (2000). Tool support for reuse of analysis patterns - a case study. In Conceptual Modeling-ER 2000 (pp. 196-209). Springer Berlin Heidelberg.

[25] Lu, S., \& Parsons, J. (2013). Ontological Rules for UML-Based Conceptual Modeling: Design Considerations and Frameworks for
Developing Efficient Information Systems: Models, Theory, and Practice: Models, Theory, and Practice, 177.

[26] Ribeiro, F. G. C., Pereira, C. E., Rettberg, A., \& Soares, M. S. (2016). Model-based requirements specification of real-time systems with UML, SysML and MARTE. Software \& Systems Modeling, 1-19.

[27] Maróti, M., Kecskés, T., Kereskényi, R., Broll, B., Völgyesi, P., Jurácz, L., ... \& Lédeczi, Á. (2014). Next Generation (Meta) Modeling: Weband Cloud-based Collaborative Tool Infrastructure. In MPM@ MoDELS (pp. 41-60).

[28] Chin Boo Soon; David C. W. (2010, May) "How Cloud Computing Changes Systems Analysis and Design" International Conference on Cloud Computing \& Virtualization; (pp. 148)

[29] Agarwal, R., \& Dhar, V. (2014). Editorial—Big data, data science, and analytics: The opportunity and challenge for IS research. Information Systems Research, 25(3), 443-448.

[30] Bentley, L. D., \& Jeffrey, L. (2007). System Analysis \& Design Methods (pp. 229-235).

[31] Dennis, A., Wixom, B. H., \& Roth, R. M. (2012). Systems analysis and design. John Wiley \& Sons (pp. 119-143). 